\title{
Diaǵnóstico e tratamento da síndrome de Sjögren
}

\author{
Sjögren's syndrome:diagnosis and treatment
}

\author{
Sergio Felberg' \\ Paulo Elias Correa Dantas ${ }^{2}$
}

Setor de Córnea e Doenças Externas do Departamento de Oftalmologia da Santa Casa de São Paulo - São Paulo (SP) - Brasil.

${ }^{1}$ Pós-graduando da Universidade Federal de São Paulo - UNIFESP - São Paulo (SP) - Brasil;

${ }^{2}$ Doutor em Medicina pela Universidade de São Paulo USP - São Paulo (SP) - Brasil.

Endereço para correspondência: Sergio Felberg. Rua Conselheiro Brotero, 1273 - São Paulo (SP) CEP 01232-011

E-mail: sergio@oftalmosantacasa.com.br

Recebido para publicação em 15.08.2005

Última versão recebida em 02.03.2006

Aprovação em 05.03.2006

Nota Editorial: Depois de concluída a análise do artigo sob sigilo editorial e com a anuência do Dr. Marcelo Carvalho da Cunha sobre a divulgação de seu nome como revisor, agradecemos sua participação neste processo.

\section{RESUMO}

A síndrome de Sjögren (SS) é doença sistêmica inflamatória crônica, de provável etiologia auto-imune, com distribuição mundial. Seu quadro clínico é responsável por considerável impacto na qualidade de vida de seus portadores. O objetivo deste artigo é descrever seus principais sintomas, os critérios atualmente usados para seu diagnóstico e as modalidades terapêuticas disponíveis até o momento.

Descritores: Síndrome de Sjögren/diagnóstico; Síndrome de Sjögren/terapia; Síndromes do olho seco; Xeroftalmia; Xerostomia; Ceratoconjuntivite seca

\section{INTRODUCূ̃̃̃O}

A síndrome de Sjögren (SS) é uma doença sistêmica inflamatória crônica, de provável etiologia auto-imune, com distribuição mundial. As glândulas lacrimais e salivares são os principais órgãos afetados pela infiltração linfo-plasmocitária, originando disfunções que desencadeiam quadro clássico de xeroftalmia (olhos secos) e xerostomia (boca seca). Outras glândulas exócrinas também podem ser acometidas como o pâncreas, glândulas sudoríparas, glândulas mucosas dos tratos respiratório, gastrointestinal e uro-genital ${ }^{(1-3)}$.

A SS pode existir como doença primária das glândulas exócrinas (SS primária) ou estar associada a outras doenças auto-imunes como artrite reumatóide, lúpus eritematoso sistêmico, esclerose sistêmica progressiva, esclerodermia, doença de Graves, dentre outras (SS secundária) ${ }^{(4)}$. Os pacientes com SS apresentam elevada incidência de linfoma maligno de células B tipo não-Hodgkin, quando comparada com a da população sadia ${ }^{(5)}$.

Fatores ambientais, como infecções virais prévias (vírus Epstein-Barr, citomegalovírus, herpes vírus humano, vírus da hepatite $\mathrm{C}$ dentre outros) ou bacterianas (Helicobacter pylori) já foram relacionadas como potenciais desencadeadores da resposta imune ao tecido glandular, devido à freqüente concomitância em pacientes com $\mathrm{SS}^{(6-7)}$. Cerca de nove mulheres são acometidas para cada homem, por este motivo, disfunções hormonais parecem fazer parte da fisiopatologia no desenvolvimento da SS, principalmente as deficiências de andrógenos, estrógeno e de progesterona ${ }^{(8-9)}$.

Há hiper-reatividade dos linfócitos B, que se convertem em plasmócitos e produzem anticorpos contra antígenos do epitélio dos ácinos e dos ductos das glândulas exócrinas. Linfócitos T supressores também são atingidos, perpetuando a atividade dos linfócitos B ativados e a agressão tecidual ${ }^{(10-11)}$.

Embora avanços recentes tenham ocorrido na elucidação da fisiopatologia da SS, muitos pontos permanecem obscuros.

\section{QUADRO CLÍNICO}

Embora pessoas de todas as idades possam ser afetadas, a doença tem 
maior incidência entre indivíduos na quarta e quinta décadas de vida, sendo as mulheres mais acometidas do que os homens ${ }^{(12-13)}$.

As manifestações correspondem à chamada "síndrome sicca", sendo as mais encontradas:

Manifestações oculares: são variadas e estão relacionadas à diminuição da secreção lacrimal. Há fraca relação entre a intensidade dos sintomas e a gravidade das alterações encontradas na superfície ocular ${ }^{(14)}$. As manifestações oculares são mais freqüentes do que as manifestações orais nas forma secundária da SS.

O espectro clínico é bastante variável, sendo que os pacientes podem desde não apresentar sintomas até queixaremse de importantes limitações nas suas atividades diárias, com piora da qualidade de vida devido à irritação ocular, sensação de corpo estranho, queimação ocular, fotofobia, "choro sem lágrimas" e turvação visual. Os sintomas costumam piorar em ambientes secos (ar condicionado, poeira, vento), durante a leitura e uso do computador ${ }^{(15-16)}$.

Questionários podem ser utilizados para auxiliar no diagnóstico do olho seco, para avaliar o impacto da doença na qualidade de vida dos portadores e para acompanhar a evolução da doença e sua resposta aos tratamentos propostos.

A deficiência na produção de lágrima pode levar à hiperemia ocular, defeitos epiteliais, aumento da quantidade de muco na superfície ocular, ceratite filamentar, úlceras de córnea e perfuração ocular nos casos mais severos.

Os testes mais utilizados no diagnóstico do olho seco e na avaliação da superfície ocular dos pacientes com cérato-conjuntivite seca são:

a) biomicroscopia: presença de debris no filme lacrimal, vasodilatação dos vasos conjuntivais, restos celulares, defeitos epiteliais e úlceras de córnea,

b) avaliação do menisco lacrimal: geralmente escasso ou ausente,

c) testes de Schirmer (basal, com anestésico e com estímulo da mucosa nasal),

d) coloração da superfície ocular com Rosa Bengala, fluoresceína ou lisamina verde,

e) tempo de ruptura do filme lacrimal,

f) teste do "clearence" do filme lacrimal,

g) medida da osmolariade da lágrima (osmolaridade aumentada),

h) avaliação do perfil protéico do filme lacrimal (diminuição das concentrações de lisozima, lactoferrina e albumina),

i) citologia de impressão: pode evidenciar alterações das células caliciformes (embora o mecanismo que leve às alterações das células caliciformes na cérato-conjuntivite seca ainda seja motivo de controvérsia, supõe-se que ocorra secundariamente à hiper-osmolaridade do filme lacrimal ou à produção de autoanticorpos diretamente contra as células caliciformes) ${ }^{(17-18)}$.

Manifestações orais: boca seca, cáries freqüentes, necessidade de umedecer a boca freqüentemente, necessidade da ingestão de líquidos durante a noite com comprometimento da qualidade do sono, dificuldade na deglutição de alimentos sólidos, dor à deglutição, aftas e úlceras na mucosa labial, infecções bucais (principalmente candidíase), aumento no volume da glândula parótida (mais freqüente na SS primária). Cintilografia salivar, sialografia da glândula parótida, ultrasonografia das glândulas salivares e a estimativa do fluxo salivar podem conduzir ao diagnóstico.

Manifestações musculo-esqueléticas: são mais freqüentes na SS secundária. Dores ósteo-articulares, fadiga e deformidades articulares.

Manifestações respiratórias: secura da mucosa nasal, falta de ar e infecções frequientes do trato respiratório são os achados mais comuns.

Manifestações genito-urinárias: secura e prurido vaginal, coito doloroso e dor à micção podem ocorrer. Pacientes com SS primária podem apresentar glomérulo-nefrites.

Manifestações cutâneas: mais freqüentes na SS secundária. Despigmentações, eritemas, prurido e eczemas.

Manifestações vasculares: fenômeno de Raynauld, trombose venosa profunda e vasculites (pele, fígado e rins).

Manifestações psiquiátricas: ansiedade, depressão e distúrbios da personalidade foram descritos com maior frequiência nos pacientes com SS que na população geral ${ }^{(1-3)}$.

\section{AUTO-ANTICORPOS CIRCULANTES}

Anticorpos órgãos-específicos descritos na SS incluem anticorpos contra antígenos presentes nos ductos glandulares, tireóide, mucosa gástrica, eritrócitos, pâncreas, próstata e células nervosas. Auto-anticorpos não específicos também podem ser encontrados como fator reumatóide (FR), fator antinúcleo (FAN), anticorpo anti-mitocondrial, anticorpo anticentrômero, dentre outros ${ }^{(19)}$.

Anticorpos contra ribonucleoproteínas: anti-Ro (SS-A) e anti-La (SS-B) são muito freqüentes nos pacientes com SS primária e menos freqüentes na SS secundária. Já o FR e FAN são mais freqüentes na SS secundária que na primária. Também estão circulantes no sangue periférico auto-anticorpos contra receptores muscarínicos (anti-MUC3) e contra proteínas do citoesqueleto das células acinares (anti-alfa-fodrim) ${ }^{(20)}$.

\section{CRITÉRIOS DIAGNÓSTICOS}

O fato de serem adotados diversos critérios diagnósticos propostos por diferentes entidades e sociedades para definir a SS, torna difícil ou quase impossível comparações entre os diversos estudos clínicos, principalmente com relação ao tratamento e aos dados epidemiológicos. Nenhum sinal, achado clínico ou imuno-marcador descrito até o momento é aceito isoladamente, como ideal para fechar o diagnóstico da SS ou detectar os períodos de atividade e remissão da doença. É importante que o paciente com suspeita de SS seja avaliado por 
equipe multidisciplinar composta de oftalmologistas, reumatologistas, otorrinolaringologistas e dentistas, dentre outros.

A demonstração de biópsia de fragmento da glândula salivar menor com evidência de infiltrado linfo-plasmocitário contendo 50 ou mais linfócitos (este conglomerado é chamado de "focus") representa um dos exames mais importante para o diagnóstico do componente oral da SS. No entanto, alguns pacientes com quadro grave de secura bucal apresentam inflamação apenas moderada nas biópsias obtidas das glândulas salivares menores, indicando que outros mecanismos além da destruição tecidual devem estar relacionados à disfunção das glândulas exócrinas.

Os critérios mais aceitos para o estabelecimento do diagnóstico da SS são:

\section{1) Critérios de San Francisco para o diagnóstico de SS primária e SS secundária $(1994)^{(21)}$ :}

-SS primária

a) Biópsia de glândula salivar menor evidenciando sialoadenite focal com infiltração linfocitária com mais de 1 "focus"/ $4 \mathrm{~mm}^{2}$ ou lesão benigna linfo-epitelial localizada em glândula salivar maior.

b) Diagnóstico de cérato-conjuntivite seca

- uso de Rosa Bengala demonstrando comprometimento córneo-conjuntival e

- redução do menisco lacrimal ou do tempo de ruptura do filme lacrimal ou

- teste de Schirmer I (sem anestésico) menor ou igual a $5 \mathrm{~mm}$ em 5 minutos

\section{-SS secundária}

a) Presença de artrite reumatóide ou outra doença do tecido conectivo

b) Pelo menos um dos itens descritos para o diagnóstico da SS primária

Possível SS: pelo menos um dos itens descritos para o diagnóstico da SS primária e uma das doenças a seguir: infiltrado linfocítico pulmonar, nefrite intersticial, púrpura, hepatite crônica (sem cirrose ou infecção), neuropatia periférica e hiper-gamaglobulinemia.

\section{2) Critérios de San Diego para o diagnóstico de SS (1986) ${ }^{(22)}$ :}

a) evidência objetiva de cérato-conjuntivite seca, documentada com coloração por Rosa Bengala ou fluoresceína.

b) evidência objetiva da diminuição do fluxo salivar.

c) biópsia de glândula salivar menor, contendo pelo menos 4 lóbulos e com presença de pelo menos 2 "foci"/4 $\mathrm{mm}^{2}$.

d) Presença de doença auto-imune sistêmica comprovada por auto-anticorpos séricos como FR, FAN, SS-A e SS-B.

SS: quando os quatro itens são encontrados

Possível SS: quando três itens estão presentes

Exclusão: doenças existentes como linfoma, infecção pelo HIV, uso de medicações que sabidamente causam olho seco, sarcoidose, doença enxerto x hospedeiro.

\section{3) Critérios europeus modificados pelo Grupo de Consen-} so Americano-Europeu (2002) ${ }^{(23)}$ :

\section{Sintomas oculares}

Pelo menos uma resposta afirmativa para uma das três questões formuladas abaixo:

a) Tem problemas oculares diários e persistentes, relacionados a quadro de olho seco há mais de três meses?

b) Tem sensação de areia ou queimação ocular?

c) Usa colírios lubrificantes mais de três vezes ao dia?

\section{Sintomas orais}

Pelo menos uma resposta afirmativa para uma das três questões formuladas abaixo:

a) Tem sensação de boca seca há mais de três meses?

b) Tem inchaço recorrente ou persistente das glândulas salivares, na idade adulta?

c) Sente necessidade de ingerir líquidos para ajudar na deglutição de alimentos sólidos?

\section{Sinais oculares}

Evidencia de modo objetivo o comprometimento ocular, quando pelo menos um dos dois testes abaixo é positivo.

a) Teste de Schirmer I ( $\leq 5 \mathrm{~mm}$ em 5 minutos)

b) Rosa Bengala ( $\geq 4$ pontos na escala de Bijsterveld)

\section{Achados histopatológicos}

Aglomeração de pelo menos 50 células mononucleares numa biópsia de $4 \mathrm{~mm}^{2}$ da glândula salivar.

\section{Comprometimento da glândula salivar}

Evidencia de modo objetivo o comprometimento das glândulas salivares, com pelo menos um dos três métodos abaixo.

a) Cintilografia da glândula salivar

b) Sialografia da glândula parótida

c) Fluxo salivar sem estímulo reflexo ( $\leq 1,5 \mathrm{~mL}$ em 15 minutos $)$

\section{Auto-anticorpos}

Presença de pelo menos um dos seguintes auto-anticorpos séricos:

a) Anticorpos contra os antígenos Ro/SS-A ou La/SS-B

b) Anticorpos anti-nuclear

c) Fator reumatóide

Critérios de exclusão: Linfoma pré-existente, AIDS, sarcoidose ou doença do enxerto $\mathrm{x}$ hospedeiro.

Provável SS primária: Presença de pelo menos 3 dos 6 itens.

SS primária: Presença de pelo menos 4 dos 6 itens (aceitando como padrão sorológico positivo apenas SS-A ou SS-B).

Provável SS secundária: Combinação da resposta positiva para os itens 1 ou 2 com pelo menos 1 item positivos entre as questões 3, 4 ou 5 .

SS secundária: Combinação da resposta positiva para os itens 1 ou 2 com pelo menos 2 itens positivos entre as questões 3,4 ou 5 . 


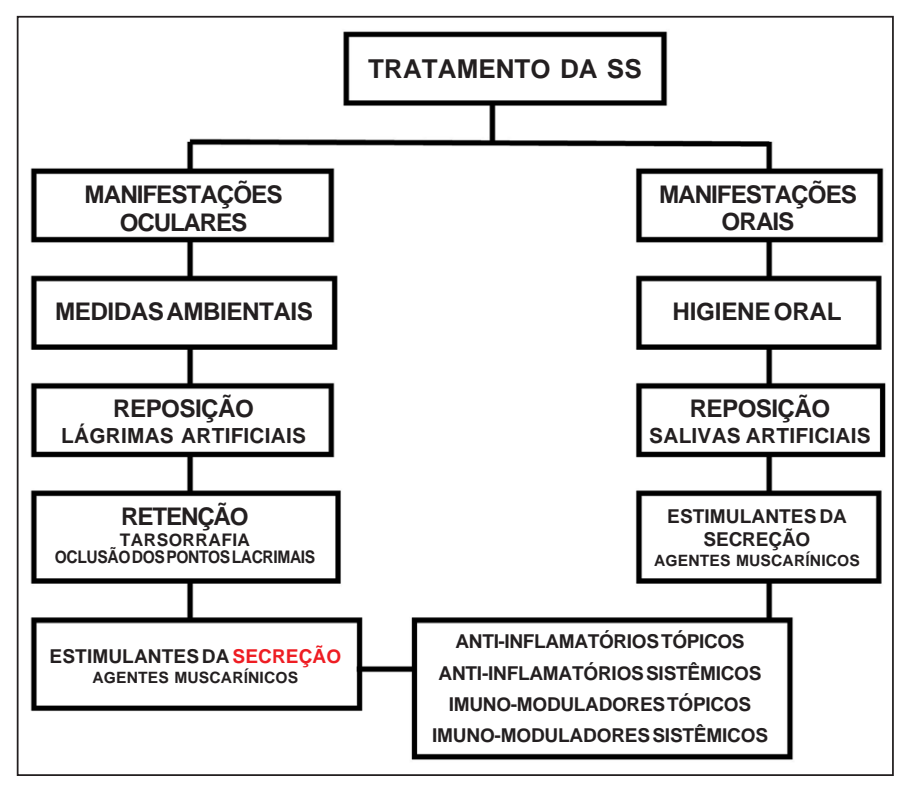

TRATAMENTO

O estabelecimento do diagnóstico da SS é fundamental para a instituição precoce do tratamento. Não há, até o momento, cura para a SS. O tratamento tem por objetivo o alívio dos sinais e sintomas, com conseqüente melhora na qualidade de vida dos pacientes, além da modificação no curso da doença a fim de que as seqüelas sejam evitadas ou minimizadas. São utilizados:

1) Tratamentos substitutivos e de retenção: administração de colírios lubrificantes sem preservantes, pomadas e géis muitas vezes são suficientes para proporcionar alívio dos sintomas oculares e prevenir complicações corneais ${ }^{(24)}$. Colírios hipotônicos ou isotônicos, à base de hialuronato de sódio mostraram-se eficazes no alívio dos sintomas ${ }^{(25)}$. Os colírios de soro autólogo contém fatores de crescimento, vitamina A e interleucinas, auxiliando na estabilização da superfície ocu$\operatorname{lar}^{(26)}$. Oclusão dos pontos lacrimais e tarsorrafia são medidas usadas para reter maior quantidade de lágrima na superfície ocular. Medidas ambientais que visam aumentar a umidade relativa do ar nos ambientes frequientados pelos portadores da SS podem ser úteis nos casos mais graves (panos úmidos, bacias com água, aquário) ${ }^{(18)}$.

Higiene oral rigorosa é importante para prevenir infecções bucais. Para alívio da xerostomia, gomas de mascar sem açúcar, água com gotas de limão, agentes mucolíticos como a bromexina e formulações de saliva artificial podem ser úteis no alívio dos sintomas ${ }^{(1,27)}$.

2) Estimulação da produção de lágrima e saliva: agonistas muscarínicos de uso oral, como a pilocarpina e a cevimelina agem nos receptores muscarínicos das glândulas estimulando a secreção salivar e lacrimal com melhora objetiva e subjetiva do quadro clínico e poucos efeitos colaterais associados ${ }^{(28-30)}$.
3) Redução da inflamação local: administração tópica de corticosteróides reduz o processo inflamatório, estabiliza a superfície ocular, melhora sinais e sintomas oculares, mas seu uso prolongado está associado a efeitos colaterais como desenvolvimento de glaucoma e catarata ${ }^{(31)}$. Ciclosporina-A tópica retarda a destruição da glândula lacrimal, promove apoptose dos linfócitos, suprime apoptose das células acinares e da conjuntiva e reduz a infiltração linfo-plasmocitária no tecido glandular. Como resultado, há aumento do lacrimejamento e alívio dos sintomas com poucos efeitos colaterais descritos ${ }^{(32)}$.

Alguns estudos demonstraram melhora nos sinais e sintomas de pacientes portadores de SS com administração tópica de andrógenos ${ }^{(33)}$.

4) Modulação da resposta imune: agentes imuno-moduladores que diminuem a intensidade da resposta imune, reduzem a linfo-proliferação e a produção dos auto-anticorpos podem estar indicados.

Dieta rica em ômegas (peixes, azeite de oliva, óleo de semente de linhaça) apresenta alguma atividade anti-inflamatória e podem melhorar a superfície ocular com algum alívio dos sintomas ${ }^{(34)}$.

A administração de baixas doses de interferon- $\alpha$ por via oral durante algumas semanas, melhorou o fluxo salivar de pacientes com SS, aliviando os sintomas da boca seca ${ }^{(35)}$.

O uso sistêmico de corticosteróides melhora os sinais e sintomas da doença, mas devido aos seus efeitos colaterais, ficam reservados para as manifestações extra-glandulares da SS. Hidroxicloroquina, ciclofosfamida e methotrexate são utilizados nos casos mais graves e de difícil controle ${ }^{(35-37)}$.

O fluxograma ao lado resume as diversas vertentes implicadas no tratamento dos pacientes portadores da SS.

\section{ABSTRACT}

Sjögren's syndrome is a systemic inflammatory autoimmune disease with worldwide distribution, responsible for considerable impact on the patient's quality of life. The aim of this article is to describe its main symptoms, the currently used different diagnostic criteria and the available treatment for the syndrome.

Keywords: Sjögren's syndrome/diagnosis; Sjögren's syndrome/therapy; Dry eye syndromes; Xerophthalmia; Xerostomia; Keratoconjunctivitis sicca

\section{REFERÊNCIAS}

1. Rehman HU. Sjogren's syndrome. Yonsei Med J. 2003;44(6):947-54. Review

2. Moutsopoulos HM, Chused TM, Mann DL, Klippel JH, Fauci AS, Frank MM, et al. Sjogren's syndrome (Sicca syndrome): current issues. Ann Intern Med. 1980;92(2 Pt 1):212-26.

3. Jonsson R, Moen K, Vestrheim D, Szodoray P. Current issues in Sjogren's syndrome. Oral Dis. 2002;8(3):130-40. Review.

4. Asmussen KH, Bowman SJ. Outcome measures in Sjogren's syndrome. Rheumatology (Oxford). 2001;40(10):1085-8. Review.

5. Asmussen K, Andersen V, Bendixen G, Benditzen K, Prause JU, Thorn J, et al. Quantitative assessment of clinical disease status in primary Sjogren's 
syndrome. A cross-sectional study using a new classification model. Scand J Rheumatol. 1997;26(3):197-205.

6. Aragona P, Magazzu G, Macchia G, Bartolone S, Di Pasquale G, Vitali C, Ferreri G. Presence of antibodies against Helicobacter pylori and its heatshock protein 60 in the serum of patients with Sjogren's syndrome. J Rheumatol. 1999;26(6):1306-11.

7. Abe T, Nakajima A, Matsunaga M, Sakuragi S, Komatsu M. Decreased tear lactoferrin concentration in patients with chronic hepatitis $\mathrm{C}$. Br J Ophthalmol. 1999;83(6):684-7.

8. Hayashi Y, Arakaki R, Ishimaru N. Apoptosis and estrogen deficiency in primary Sjogren syndrome. Curr Opin Rheumatol. 2004;16(5):522-6.

9. Hansen A, Lipsky PE, Dorner T. New concepts in the pathogenesis of Sjogren syndrome: many questions, fewer answers. Curr Opin Rheumatol. 2003; 15(5):563-70.

10. Yamamoto K. Pathogenesis of Sjogren's syndrome. Autoimmun Rev. 2003;2 (1):13-8.

11. Esch TR. Pathogenetic factors in Sjogren's syndrome: recent developments. Crit Rev Oral Biol Med. 2001;12(3):244-51.

12. Bowman SJ, Pillemer S, Jonsson R, Asmussen K, Vitali C, Manthorpe R, Sutcliffe N. Contributors to and participants at the workshop. Revisiting Sjogren's syndrome in the new millenium: perspectives on assessment and outcome measures. Report of a workshop held on 23 March 2000 at Oxford, UK. Rheumatology (Oxford). 2001;40(10):1180-8.

13. Rozman B, Novljan MP, Hocevar A, Ambrozic A, Zigon P, Kveder T, Tomsic M. Epidemiology and diagnostics of primary Sjogren's syndrome. Reumatizam. 2004;51(2):9-12. Review.

14. Hay EM, Thomas E, Pal B, Hajeer A, Chambers H, Silman AJ. Weak association between subjective symptoms or and objective testing for dry eyes and dry mouth: results from a population based study. Ann Rheum Dis. 1998;57(1):20-4.

15. Kassan SS, Moutsopoulos HM. Clinical manifestations and early diagnosis of Sjogren syndrome. Arch Intern Med. 2004;164(12):1275-84.

16. Kalk WW, Mansour K, Vissink A, Spijkervet FK, Bootsma H, Kallenberg CG, et al. Oral and ocular manifestations in Sjogren's syndrome. J Rheumatol. 2002;29(5):924-30.

17. Nishiwaki-Dantas MC. Olho seco. Arq Bras Oftalmol. 2000;62(1):101-5.

18. Nichols KK, Nichols JJ, Lynn Mitchell G. The relation between tear film tests in patients with dry eye disease. Ophthalmic Physiol Opt. 2003;23(6):553-60.

19. Toda I. Autoantigens and Sjogren syndrome. Cornea. 2002;21(2 Suppl 1): S13-6. Review.

20. Harley JB, Alexander EL, Bias WB, Fox OF, Provost TT, Reichlin M, et al. Anti-Ro (SS-A) and anti-La (SS-B) in patients with Sjogren's syndrome. Arthritis Rheum. 1986;29(2):196-206.

21. Daniels TE, Whitcher JP. Association of patterns of labial salivary gland inflammation with keratoconjunctivitis sicca. Analysis of 618 patients with suspected Sjogren's syndrome. Arthritis Rheum. 1994;37(6):869-77.

22. Fox RI, Robinson CA, Curd JG, Kozin F, Howell FV. Sjogren's syndrome. Proposed criteria for classification. Arthritis Rheum. 1986;29(5):577-85.

23. Vitali C, Bonbardieri S, Jonsson R, Moutsopoulos HM, Alexander EL,
Carsons SE, Daniels TE, Fox PC, Kassan SS, Pillemer SR, Talal N, Weisman MH; European Study Group on Classification Criteria for Sjögren's Syndrome. Classification criteria for Sjogren's syndrome: a revised version of the European criteria proposed by the American-European Consensus Group. Ann Rheum Dis. 2002;61(6):554-8. Review.

24. Fox RI, Michelson P. Approaches to the treatment of Sjogren's syndrome. J Rheumatol Suppl. 2000;61:15-21. Review.

25. Aragona P, Di Stefano G, Ferreri F, Spinella R, Stilo A. Sodium hyaluronate eye drops of different osmolarity for the treatment of dry eye in Sjogren's syndrome patients. Br J Ophthalmol. 2002;86(8):879-84.

26. Noble BA, Loh RS, MacLennan S, Pesudovs K, Reynolds A, Bridges LR, et al. Comparison of autologous serum eye drops with conventional therapy in a randomised controlled crossover trial for ocular surface disease. $\mathrm{Br} \mathrm{J}$ Ophthalmol. 2004;88(5):647-52.

27. Derk CT, Vivino FB. A primary care approach to Sjogren's syndrome. Helping patients cope with sicca symptoms, extraglandular manifestations. Postgrad Med. 2004;116(3):49-54,59,65.

28. Ono M, Takamura E, Shinozaki K, Tsumura T, Hamano T, Yagi Y, Tsubota K. Therapeutic effect of cevimeline on dry eye in patients with Sjogren's syndrome: a randomized, double-blind clinical study. Am J Ophthalmol. 2004;138(1):6-17.

29. Nelson JD, Friedlaender M, Yeatts RP, Yee R, McDermott M, Orlin S, et al. Oral pilocarpine for symptomatic relief of keratoconjunctivitis sicca in patients with Sjogren's syndrome. The MGI PHARMA Sjogren's Syndrome Study Group. Adv Exp Med Biol. 1998;438:979-83.

30. Petrone D, Condemi JJ, Fife R, Gluck O, Cohen S, Dalgin P. A doubleblind, randomized, placebo-controlled study of cevimeline in Sjogren's syndrome patients with xerostomia and keratoconjunctivitis sicca. Arthritis Rheum. 2002;46(3):748-54.

31. Avunduk AM, Avunduk MC, Varnell ED, Kaufman HE. The comparison of efficacies of topical corticosteroids and nonsteroidal anti-inflammatory drops on dry eye patients: a clinical and immunocytochemical study. Am J Ophthalmol. 2003;136(4):593-602.

32. Gunduz K, Ozdemir O. Topical cyclosporin treatment of keratoconjunctivitis sicca in secondary Sjogren's syndrome. Acta Ophthalmol (Copenh). 1994;72 (4):438-42.

33. Sullivan DA, Sullivan BD, Evans JE, Schirra F, Yamagami H, Liu M, et al. Androgen deficiency, Meibomian gland dysfunction, and evaporative dry eye. Ann N Y Acad Sci. 2002;966:211-22.

34. Brown NA, Bron AJ, Harding JJ, Dewar HM. Nutrition supplements and the eye. Eye. 1998;12(Pt 1):127-33.

35. Ferraccioli GF, Salaffi F, De Vita S, Casatta L, Avellini C, Carotti M, et al. Interferon alpha-2 (IFN alpha 2) increases lacrimal and salivary function in Sjogren's syndrome patients. Preliminary results of an open pilot trial versus OH-chloroquine. Clin Exp Rheumatol. 1996;14(4):367-71.

36. Sheppard JD. Guidelines for the treatment of chronic dry eye disease. Manag Care. 2003;12(12 Suppl):20-5.

37. Fox RI. Sjogren's syndrome: current therapies remain inadequate for a common disease. Expert Opin Investig Drugs. 2000;9(9):2007-16. 